\title{
ATUAÇÃO DO PROFISSIONAL DE FISIOTERAPIA NA RESIDÊNCIA MULTIPROFISSIONAL EM URGÊNCIA E TRAUMA: RELATO DE EXPERIÊNCIA
}

PHYSICAL THERAPY PRACTITIONER'S WORK IN THE MULTIPROFESSIONAL RESIDENCY IN URGENCY AND TRAUMA: EXPERIENCE

ACTUACIÓN DEL PROFESIONAL DE FISIOTERAPIA EN LA RESIDENCIA MULTIPROFESIONAL EN URGENCIA Y TRAUMA: INFORME DE

1. Fisioterapeuta. Residente no Programa de Urgência e Trauma da Faculdade de Educação e Cultura de Vilhena (FAEV/UNESC). E-mail: line.ibna@gmail.com ORCID: https://orcid.org/0000-0002-9970-4766

2. Fisioterapeuta. Residente do Programa de Urgência e Trauma da FAEV/UNESC.E-mail: michelelupatini@ hotmail.com ORCID: https://orcid.org/0000-0001-6555-1645

3. Fisioterapeuta. Coordenadora do Programa de Residência Multiprofissional em Saúde da FAEV/UNESC. E-mail: prof.aline@unescnet.br ORCID: https://orcid.org/0000-0001-9041-4773 


\section{ABSTRACT}

The multiprofessional residency in health has spread throughout Brazil and drawn the attention of many professionals to work in the Brazilian National Health System (Sistema Único de Saúde [SUS]). These professionals, through experiences, receive qualification and training to work by having the SUS's healthcare practices as a basis. The physical therapist is a member of the multiprofessional team who has a wide range of activities in the hospital environment, from emergency care at the emergency room (ER) to rehabilitation at inpatient sectors and intensive care units (ICUs). This study describes the experiences of a resident in physical therapy as a member of the multiprofessional team in urgency and trauma and the scope of her work in the hospital environment. Through the experience report of this resident in physical therapy, the routines, professional practices, difficulties faced, and care in the domain of multiprofessional residency in health are addressed. This program contributes decisively to health practitioners' training, however, there is still a lack of studies that report the experiences and the scope of a physical therapy practitioner's work in hospital care focused on urgency and trauma.

\section{RESUMEN}

La residencia multiprofesional en salud se ha extendido por todo Brasil y ha llamado la atención de muchos profesionales para actuar en el Sistema Único de Salud Brasileño (Sistema Único de Saúde [SUS]). Estos profesionales, a través de vivencia, reciben calificación y entrenamiento para actuar al tener como base las prácticas de atención del SUS. El fisioterapeuta es un miembro del equipo multiprofesional que tiene una amplia gama de actividades en el ambiente hospitalario, desde atención de emergencia en la sala de urgencias (SU) hasta rehabilitación en sectores de hospitalización y unidades de cuidados intensivos (UCI). Este estudio describe las experiencias de una residente en fisioterapia como miembro del equipo multiprofesional en urgencia y trauma y el alcance de su trabajo en el ambiente hospitalario. A través del informe de experiencia de esta residente en fisioterapia, se abordan las rutinas, las prácticas profesionales, las dificultades enfrentadas y la atención en el ámbito de la residencia multiprofesional en salud. Este programa contribuye decisivamente a la formación de los profesionales de la salud, sin embargo, todavía hay una escasez de estudios que reporten las experiencias y el alcance del trabajo de un profesional de fisioterapia en la atención hospitalaria centrada en la urgencia y el trauma.

\section{INTRODUÇÃO}

A residência em área profissional da saúde, instituída pela Lei n. 11.129/2005, é definida como uma modalidade de pós-graduação lato sensu pautada pela educação em serviço de profissionais das diversas categorias do setor saúde (exceto a médica). Nesse sentido, com base nos princípios do Sistema Único de Saúde (SUS), a residência multiprofissional em urgência e trauma integra a formação profissional em saúde no ambiente hospitalar ${ }^{1}$.

Considerando o art. 200 da Constituição Federal de 1988 (CF/1988), que evidencia a responsabilidade do SUS na capacitação de seus profissionais, criaramse estratégias para habilitá-los a atuar em rede interdisciplinar e multidisciplinar de saúde. Desse modo, os programas de residência multiprofissional em saúde têm contribuindo com a formação desse pessoal com vistas a proporcionar o atendimento humanizado garantido por lei aos usuários do SUS ${ }^{1-3}$.

0 Programa de Residência Multiprofissional em Saúde da Faculdade de Educação e Cultura de Vilhena (FAEV/UNESC) teve início em março de
2015. As categorias profissionais envolvidas são: a) enfermagem; b) fisioterapia; c) psicologia; d) serviço social; e e) farmácia (incluindo farmácia bioquímica). E esse programa se divide em 3 residências específicas: a) residência em urgência e trauma; b) residência em enfermagem obstétrica; e c) residência em saúde mental.

Nesse contexto, o fisioterapeuta se insere na residência em urgência e trauma. A inclusão desse residente nos setores de emergência e nas unidades de terapia intensiva (UTIs) se baseia na reestruturação dos modelos de saúde brasileiros: demanda-se tanto o conhecimento sobre as políticas do hospital como acerca do perfil e das rotinas do local específico ${ }^{1}$.

Por razões conceituais, a fisioterapia surgiu como uma profissão exclusivamente reabilitadora de sequelas, mas tem evoluído na reestruturação dos modelos de saúde com base nos princípios de atenção integral e humanização da assistência do SUS. Por exemplo: estudos sinalizam a importância de aprimorar a fisioterapia motora no ambiente hospitalar, uma vez que está aliada à fisioterapia 
respiratória em certas patologias que acometem 0 sistema motor, o que pode diminuir a permanência hospitalar e aumentar os índices de recuperação após a alta ${ }^{2-3}$.

A prática da fisioterapia hospitalar se destaca ao minimizar os efeitos deletérios da inatividade do paciente acamado, reduzindo a taxa de mortalidade, a taxa de infecção e o tempo de permanência na UTI, além das complicações pós-operatórias. Vale ressaltar, ainda, os aspectos envolvidos no suporte ventilatório (invasivo ou não invasivo) aos pacientes que carecem dessa assistência nos diversos setores hospitalares ${ }^{4-5}$.

A inserção do profissional de fisioterapia na urgência e emergência hospitalar reflete sua capacidade de agregar atividades, de discutir casos com a equipe interdisciplinar e de potencializar o cuidado integral e humanizado voltado ao paciente e à sua família 5 .

As pesquisas envolvendo a atuação do profissional de fisioterapia no ambiente hospitalar ainda são escassas, o que sugere a realização de estudos que avaliem os impactos da intervenção fisioterapêutica nos setores de urgência e emergência ${ }^{6}$.

Daí surgiu nosso interesse em elaborar este artigo, que descreve a experiência de uma fisioterapeuta como membro da equipe de residência multiprofissional em urgência e trauma, relatando a abrangência de sua atuação hospitalar.

\section{METODOLOGIA}

Este estudo consiste em um relato de experiência, baseado na rotina de uma profissional de fisioterapia na residência multiprofissional em urgência e trauma, realizada entre março de 2016 e março de 2018 (2 anos).

$A$ residência multiprofissional em urgência e trauma da FAEV/UNESC, em parceria com a Secretaria Municipal de Saúde de Vilhena (RO), tem como cenário prático o Hospital Adamastor de Oliveira (Hospital Regional de Vilhena - HRV), que conta com cerca de 180 leitos distribuídos entre os seguintes setores: a) pronto-socorro (PS); b) clínica médica masculina (CMM); c) clínica médica feminina (CMF); d) ortopedia; e) UTI; f) pediatria; g) obstetrícia (maternidade); e h) unidade de terapia intensiva neonatal (UTIN). 0 HRV realiza cerca de 250 atendimentos diários em seu PS, além dos cuidados oferecidos aos pacientes já internados em suas alas; em atividade desde o início dos anos 1990, trata-se

\section{As pesquisas \\ envolvendo a atuação \\ do profissional de \\ fisioterapia no ambiente \\ hospitalar ainda são \\ escassas...}

de instituição de referência no Cone Sul do Estado de Rondônia.

\section{RESULTADOS}

Este relato de experiência recorre a: a) expressões e provocações observadas nas práticas hospitalares; b) diálogos situados no ambiente hospitalar; e c) pesquisa bibliográfica.

Cumprindo uma jornada hospitalar de 60 horas semanais, sendo 12 horas diárias (das 7:00 às 19:00), o residente de fisioterapia atua por meio de rodízio nos setores atribuídos a ele, mediante distribuição pela tutoria ao longo de seus 2 anos de permanência no programa: a) UTI; b) CMM; c) CMF; d) pediatria; e) PS; e f) UTIN. Tal jornada possibilita articular a educação em saúde às diversas profissões, às comunidades e à população geral, proporcionando o aprendizado de conteúdos técnicos, políticos e científicos e uma formação crítico-reflexiva, com troca de experiências sobre os diversos tipos de problemas de saúde ${ }^{3}$.

Cada setor hospitalar foi apresentado à residente enfocada neste estudo pelo tutor no início de cada rodizio, bem como a equipe multiprofissional que 0 compõe, suas rotinas, suas práticas e seus protocolos. Após a apresentação do setor de atuação, coube à residente elaborar seu plano de ação (um documento que descrevia o planejamento das atividades a realizar durante sua permanência no setor), que devia ser entregue mensalmente ao tutor, além do relatório mensal com as atividades desenvolvidas, com embasamento teórico e abordagem crítica dos temas levantados por ela.

Com isso em vista, havia no plano de ação: a) recebimento do plantão junto à equipe do setor; b) leitura de prontuários para identificar pacientes com prescrição de fisioterapia; c) leitura de exames complementares; d) atendimento aos pacientes; e) evolução em prontuários; e f) estudo de casos 
clínicos junto à equipe do setor.

Assim, a residente se inseria no setor e desempenhava as atribuições de sua formação profissional, sob a supervisão tanto de um preceptor ( $p$ rofissional do setor de atuação), no sentido de sanar dúvidas relativas às rotinas e práticas específicas, como de seu tutor na residência multiprofissional em saúde.

No cronograma de residência enfocado neste estudo, os primeiros setores de atuação foram as clínicas médicas (CMM e CMF), ao longo de um período de 4 meses, como se descreve a seguir.

\section{Fisioterapia em Clínicas Médicas}

As clínicas médicas (CMM e CMF) são setores de internação hospitalar nas quais os pacientes permanecem para tratamento de patologias diversas. Geralmente, os quartos são divididos por patologias: a) pneumopatias; b) pré ou pós-cirúrgicos; c) cardiopatias; d) enteropatias; e e) neuropatias (pacientes pós-acidente vascular cerebral [AVC]). Além disso, há quartos de isolamento e os reservados a outros tipos de diagnóstico. Há cerca de 40 leitos por clínica médica.

Uma equipe multiprofissional atua em esquema de escala e é composta por médicos plantonistas, enfermeiros, técnicos de enfermagem, fisioterapeuta e fonoaudiólogo, contando com o suporte do serviço social e da psicologia (quando necessário) no setor.

Durante o período em que a residente enfocada neste estudo permaneceu no setor, pôde desempenhar diversas atividades além do atendimento aos pacientes, com destaque para: a) ginástica laboral (3 vezes por semana), realizada com a equipe após o recebimento do plantão, antecedendo as atividades da equipe de enfermagem; e b) palestras sobre o vírus H1N1 (apresentando os meios de contaminação, vacinação e prevenção) para crianças, adolescentes e jovens, realizadas em horário noturno em 2 escolas da cidade.

Em relação à assistência aos pacientes, após a leitura dos prontuários e a verificação das prescrições de fisioterapia, a residente pôde realizar atendimentos voltados a patologias gerais, sendo mais frequentes as pneumopatias: a) doença pulmonar obstrutiva crônica (DPOC); b) pneumonia; c) gripe H1N1 (tendo em vista que em 2016 houve casos confirmados no hospital); d) tuberculose; e) bronquite; f) derrame pleural; e g) crise asmática, dentre outras. Também vale destacar os comprometimentos neurológicos:

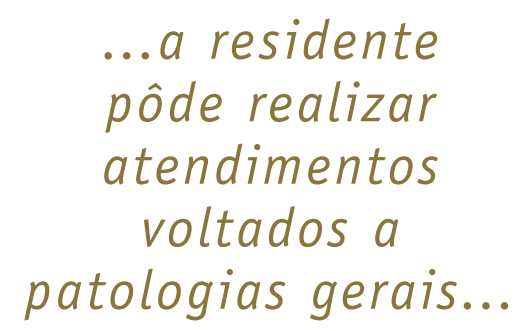

a) AVC e suas complicações (em alguns casos se mostrava necessária a aspiração de vias aéreas); b) paralisia cerebral; e c) paraplegia. E, ainda, havia as condições pós-cirúrgicas e os traumas ortopédicos, além de assistência à equipe multiprofissional para procedimentos de intubação e reanimação durante paradas cardiorrespiratórias (PCR) de pacientes da CMM e CMF ${ }^{3}$.

Um dos maiores desafios de inserir-se em um ambiente hospitalar para a prestação de serviços de fisioterapia consiste em lidar com patologias diversas, o que requer constante atualização sobre casos clínicos diferentes para proceder adequadamente diante de cada demanda. Assim, no decorrer dos meses de atuação da residente em cada setor, aulas e seminários multiprofissionais relativos a patologias e casos clínicos proporcionaram embasamento teórico à atuação prática, combinados a diálogos com os preceptores e tutores.

Acrescente-se a isso as dificuldades enfrentadas pela residente em cada setor de atuação, como a precariedade dos recursos materiais disponíveis e a falta de equipamentos necessários à assistência, o que a levou a recorrer à improvisação no atendimento aos pacientes. $E$, em alguns casos, a falta de conhecimento por parte da equipe do setor sobre a assistência fisioterapêtica ${ }^{3-5}$ demandou explicações da residente durante a prestação de alguns serviços.

\section{Fisioterapia em Unidade de Terapia Intensiva}

Esse setor constituiu o segundo momento de atuação da residente, no decorrer de 5 meses, prestando assistência junto à equipe multiprofissional local, composta por médico, enfermeiro, técnicos de enfermagem e fisioterapeuta. Em momentos específicos, ela contava com o apoio de psicólogo, fonoaudiólogo, farmacêutico e serviço social no atendimento aos pacientes ${ }^{3-4}$.

No período de permanência na UTI, durante um mês, a residente também atuou em horário vespertino no PS, atendendo emergências relativas a patologias 
respiratórias e auxiliando em procedimentos de reanimação em $P C R$, dentre outros casos diversos.

Assim como no setor anterior, a residente foi apresentada à equipe, à rotina e às práticas diárias da UTI, elaborando seu plano de ação para a assistência no setor, onde caberia a ela: a) recebimento do plantão; b) organização dos equipamentos de fisioterapia (bandeja com materiais de aspiração do paciente); c) leitura de prontuários dos pacientes; d) interação com a equipe multiprofissional na discussão de casos clínicos; e) atendimento aos pacientes; f) pesquisa sobre patologias para atender a demandas específicas dos pacientes; e g) relatório diário dos atendimentos nos prontuários e no livro de fisioterapia.

Durante o período de atuação na UTI, além das atividades de assistência aos pacientes, a residente, junto com seu preceptor, confeccionou um protocolo de fórmulas de ventilação mecânica para uso da equipe do setor e uma ficha de mapa ventilatório dos pacientes sob ventilação mecânica invasiva (VMI), para o acompanhamento de sua evolução respiratória ${ }^{6-8}$.

A UTI se mostrou um campo fértil para a aquisição de conhecimentos práticos em fisioterapia, pois a assistência fisioterapêutica é mais abrangente nesse setor devido às distintas patologias que demandam cuidados, além da participação mais frequente em procedimentos de intubação, reanimação, intervenção cardiorrespiratória, suporte respiratório (mediante ventilação mecânica invasiva e não invasiva), assistência ao paciente acamado e estimulação da retirada precoce do paciente do leito.

Visitas leito a leito foram realizadas diariamente na UTI junto com a equipe (visita multiprofissional), onde se reuniam todos os profissionais do setor e se abordavam os casos clínicos de modo individual, bem como os objetivos, as metas e as condutas do tratamento baseado no diagnóstico de cada paciente, o que se mostrou de grande valia para a aquisição de conhecimentos advindos não só da prática profissional, mas de outras categorias profissionais.

\section{Fisioterapia em Pediatria e Unidade de Terapia Intensiva Neonatal}

A pediatria e a UTIN constituem setores hospitalares distintos: enquanto a UTIN abriga recém-nascidos (RNs) de 0 até 28 dias de vida, a pediatria atende pacientes a partir dos 29 dias de vida até os 12 anos de idade.

\section{A UTI se mostrou \\ um campo fértil \\ para a aquisição de \\ conhecimentos práticos \\ em fisioterapia...}

$\mathrm{Na}$ UTIN, a equipe multiprofissional dispõe de médico pediatra, fisioterapeuta, enfermeiro, fonoaudiólogo e técnicos de enfermagem, contando, ainda, com o suporte da psicologia e do serviço social para determinadas demandas.

Dentre as condutas adotadas tanto na pediatria quanto na UTIN, o fisioterapeuta atende pacientes com sinais de esforço respiratório e proporciona manutenção do suporte respiratório mediante a oxigenoterapia.

No que se refere às práticas da residente, seguiuse o mesmo padrão dos outros setores, tendo em vista que a reabilitação fisioterapêutica não se altera consideravelmente: atuando simultaneamente em dois setores, a residente permanece na UTIN e passa para prestar assistência na pediatria pela manhã e à tarde em horários determinados (após a visita do médico plantonista para as prescrições e os pedidos de exames). Devido ao fato da UTIN receber pacientes recém-nascidos, em determinados casos se mostrava necessária a presença da fisioterapeuta para intervenções durante o parto ou para lidar com broncoaspiração durante a amamentação $0^{6,8-11}$.

No geral, as patologias com prescrição de fisioterapia na ala pediátrica e na UTIN eram relativas ao trato respiratório: a) pneumonia; b) bronquite; c) bronquiolite; d) derrame pleural; e e) crise asmática. Em alguns casos, além dos procedimentos de rotina, mostrava-se necessária a aspiração de via aéreas, por exemplo, em pacientes com paralisia cerebral acometidos com pneumonia por broncoaspiração $0^{4,9}$.

Além da fisioterapia respiratória, realizavase o atendimento de fisioterapia motora junto a pacientes com patologias neurológicas (paralisia cerebral) e traumas ortopédicos, bem como pacientes com acometimento motor decorrente de lesões por acidente ofídico (picada de cobras e animais peçonhentos) que demandavam reabilitação. Em relação à UTIN, de modo específico, as patologias com prescrição de atendimento fisioterapêutico eram: a) doença da membrana hialina; b) síndrome 
de aspiração de mecônio; c) prematuridade; d) pneumonia; e e) infecções neonatais ${ }^{6,8-9}$.

Vale destacar, em meio às atividades da residente durante sua permanência na UTIN, a criação e a implantação do projeto "Polvo de Amor", no qual, após a autorização da direção hospitalar e o treinamento por parte da equipe do setor, fez-se uso do polvo de crochê (devidamente confeccionado e esterilizado) junto a recém-nascidos com critérios específicos de prematuridade, baixo peso e irritabilidade, dentre outros avaliados pela equipe neonatal, contando com boa aceitação tanto da equipe multiprofissional como dos pais dos RNs.

Assim como nos demais setores, além do atendimento específico aos pacientes, a residente atuou em conjunto com a equipe multiprofissional, realizando atividades de ginástica laboral com os funcionários hospitalares, sessões de capacitação e palestras sobre patologias observadas no setor, visando à atualização dessa equipe acerca dos procedimentos de oxigenoterapia neonatal e pediátrica.

\section{CONCLUSÃO}

A residência multiprofissional tem contribuído com a formação de profissionais da saúde que buscam qualificação e capacitação. Ao atuar no ambiente hospitalar, eles passam a conhecer em maior profundidade as práticas assistenciais do SUS e as rotinas de urgência e trauma, proporcionando assistência integral e humanizada aos usuários dos serviços de saúde.

Os desafios do programa de residência da FAEV/ UNESC englobaram: a) a falta de recursos materiais observada em alguns setores de atuação, que comprometia a qualidade da assistência em alguns casos; b) a falta de conhecimento por parte de alguns profissionais de alguns setores de atuação acerca do papel da residente (profissional formada que buscava maior qualificação); e c) a falta de conhecimento por parte da equipe multiprofissional sobre a abrangência da atuação da fisioterapeuta no ambiente hospitalar.

Nesse sentido, a interação com a equipe multiprofissional durante a residência em saúde proporcionou conhecimentos práticos à residente para lidar de modo adequado com problemas dessa natureza.

Em suma, pode-se ressaltar a necessidade de disponibilizar recursos humanos e materiais

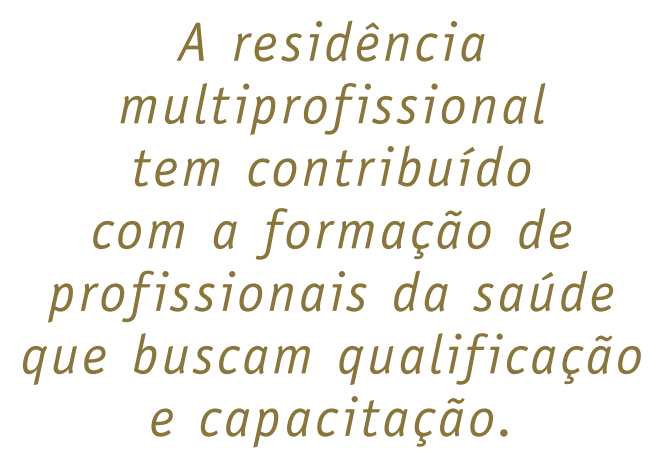

adequados, de modo que os residentes em saúde ajudem a proporcionar aos usuários do SUS a assistência integral e humanizada exigida pela legislação brasileira. E também se constata a necessidade de novos estudos que abordem a atuação do residente em fisioterapia como membro da equipe multiprofissional no ambiente hospitalar.

\section{CONTRIBUIÇÃO DAS AUTORAS}

Allynne Bispo de Freitas Pereira contribuiu com a realização da pesquisa, o delineamento do estudo e a redação do manuscrito. Michele Lupatini Ferreira contribuiu com o delineamento do estudo e a redação do manuscrito. Aline Fernanda Barbosa Bernardo contribuiu com o delineamento do estudo e a revisão crítica do manuscrito.

\section{REFERÊNCIAS}

1. Brasil. Residência multiprofissional em saúde: experiências, avanços e desafios. Brasília (DF): Ministério da Saúde; 2006.

2. Nascimento DDG, Oliveira MAC. A Política de Formação de Profissionais da Saúde para o SUS: considerações sobre a Residência Multiprofissional em Saúde da Família. REME Rev Min Enferm [serial on the internet]. 2006 [cited 2018 Apr 12];10(4):4359. Available from: http://pesquisa.bvs.br/brasil/ resource/pt/bde-16243

3. Mesquita CRS, Bastos VPD. Desafios da atuação fisioterapêutica no contexto da residência multiprofissional: relato de experiência. Journal of Health Connections [serial on the internet]. 2017 [cited 2018 Apr 15];1(1):19-32. Available from: http://revistaadmmade.estacio.br/index.php/ journalhc/article/view/3321/1800

4. Paiva DRD, Guimarães VS, Rôla QCS, Castro IPRD, Gomes KS, Anjos JLM. Inserção e atuação de fisioterapeutas residentes em um serviço de emergência hospitalar: relato de experiência. Rev Pesqui Fisioter [serial on the internet]. 2017 [cited 
2018 Apr 21];7(2):255-60. Available from: https:// www5.bahiana.edu.br/index.php/fisioterapia/ article/viewFile/1351/875

5. Piccoli A, Werle RW, Kutchak F, Rieder MDM. Indicações para inserção do profissional fisioterapeuta em uma unidade de emergência. ASSOBRAFIR Ciência [serial on the internet]. 2013 [cited $2018 \mathrm{Apr} 22$ ];4(1):33-41. Available from: http://www.uel.br/revistas/uel/index.php/rebrafis/ article/view/13412/12758

6. Alves NA. A importância da atuação do fisioterapeuta no ambiente hospitalar. Ensaios e Ciência: Ciências Biológicas, Agrárias e da Saúde [serial on the internet]. 2012 [cited 2018 Apr 21];16(6):173-84. Available from: http:// pgsskroton.com.br/seer/index.php/ensaioeciencia/ article/viewFile/2750/2607

7. Parente JRF. Preceptoria e tutoria na Residência Multiprofissional em Saúde da Família. Sanare (Sobral, 0nline) [serial on the internet]. 2008 [cited 2018 Apr 12];7(2):47-53. Available from: https:// sanare.emnuvens.com.br/sanare/article/view/31

8. Silva Neto BG, Souza DPM. A prática do fisioterapeuta intensivista junto à equipe multidisciplinar [document on the internet]. 2012 [cited 2018 Apr 22]. Available from: http:// portalbiocursos.com.br/ohs/data/docs/27/03 - A prYtica do fisioterapeuta intensivista junto $Y$ equipe multidisciplinar.pdf

9. Cordeiro AL, Lima TG. Fisioterapia em unidades de emergência: uma revisão sistemática. Rev Pesqui Fisioter [serial on the internet]. 2017 [cited 2018 Apr 18];7(2):276-81. Available from: https://www5. bahiana.edu.br/index.php/fisioterapia/article/ view $/ 1360$

10. Alemira ICN, Lima GM, Costa LA, Carneiro LM, Santos MIG, Macêdo RC et al. Atuação da fisioterapia na urgência e emergência de um hospital referência em trauma e queimados de alta e média complexidade. Revista da Universidade Vale do Rio Verde [serial on the internet]. 2017 [cited 2018 Apr 18];15(1):791805. Available from: http://periodicos.unincor.br/ index.php/revistaunincor/article/view/3490

11. Mariano LCO, Marinho TPC. Residência multiprofissional em saúde na perspectiva do serviço social em hospital público: relato de experiência. Sanare (Sobral, Online) [serial on the internet]. 2017 [cited 2018 Apr 10];16(1):136-42. Available from: https://sanare.emnuvens.com.br/sanare/ article/view/1105
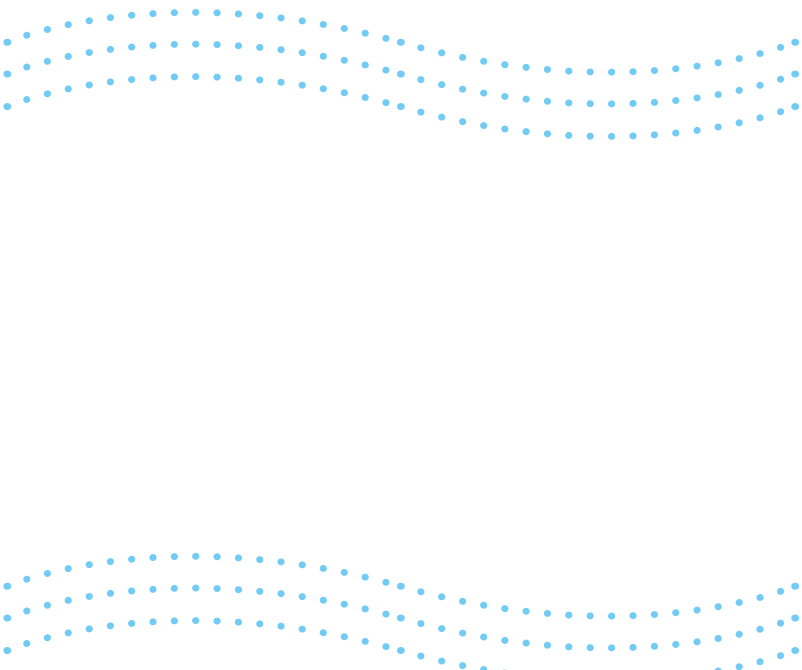
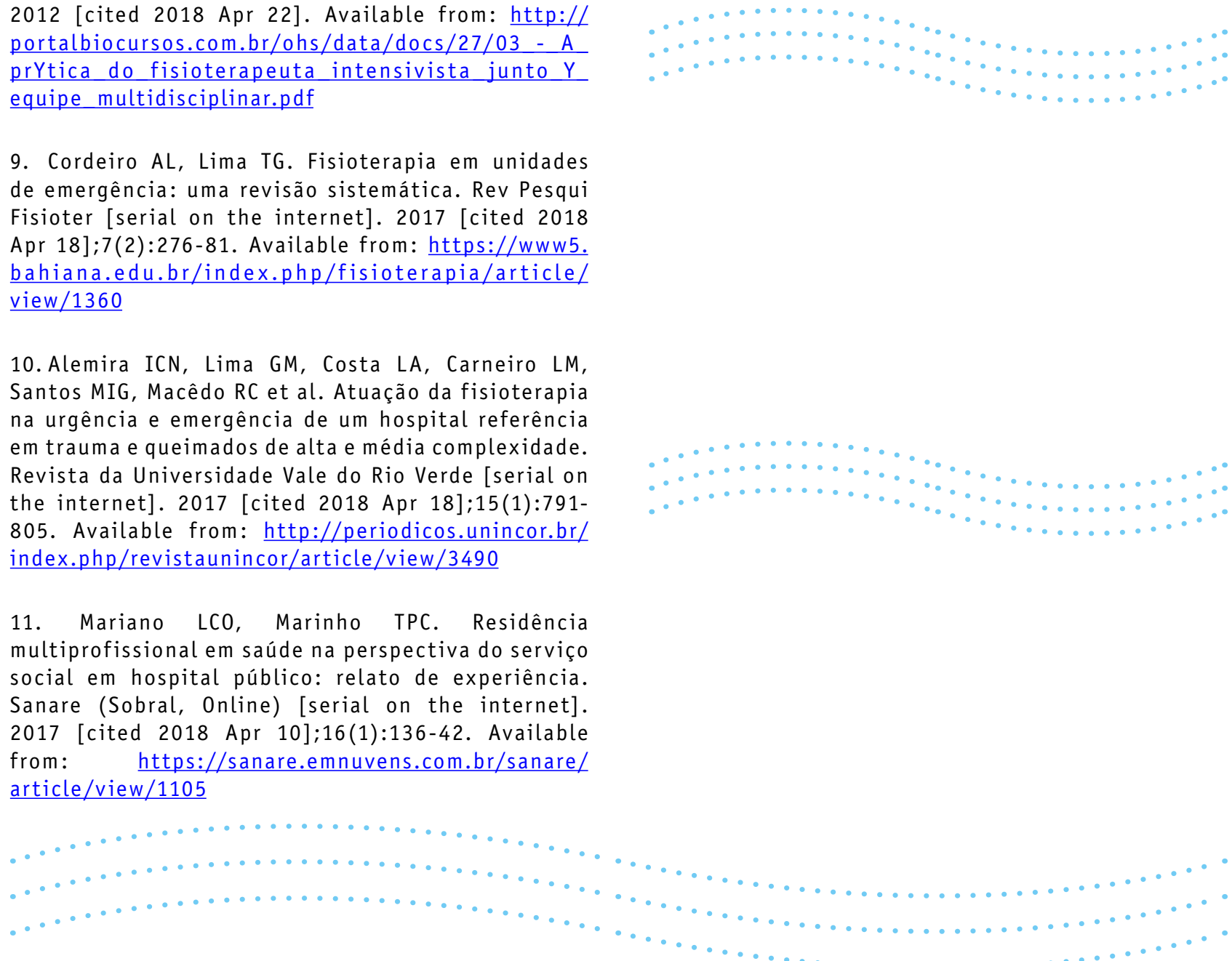